\title{
1 Over-expression of progestin and adipoQ receptor family member 4 in human endometrial cancer.
}

Shahan Mamoor, $\mathrm{MS}^{1}$

${ }^{1}$ shahanmamoor@gmail.com

East Islip, NY USA

Gynecologic cancers including cancers of the endometrium are a clinical problem ${ }^{1-4}$. We mined published microarray data ${ }^{5,6}$ to discover genes associated with endometrial cancers by comparing transcriptomes of the normal endometrium and endometrial tumors from humans. We identified progestin and adipoQ receptor family member 4, encoded by PAQR4, as among the most differentially expressed genes, transcriptome-wide, in cancers of the endometrium. PAQR4 was expressed at significantly higher levels in endometrial tumor tissues as compared to the endometrium. Importantly, in human endometrial cancer, primary tumor expression of PAQR4 was correlated with overall survival in black patients with low mutational burden. PAQR4 may be a molecule of interest in understanding the etiology or progression of human endometrial cancer.

Keywords: endometrial cancer, gynecologic cancers, endometrium, PAQR4, progestin and adipoQ receptor family member 4 , systems biology of endometrial cancer, targeted therapeutics 
Endometrial cancer is the most common gynecologic cancer in the developed world ${ }^{1}$. Over the last three decades, the incidence of endometrial cancer has increased $21 \%{ }^{4}$ and the death rate has increased $100 \%{ }^{3}$. We harnessed the power of independently published microarray datasets $^{5,6}$ to determine in an unbiased fashion and at the systems-level genes most differentially expressed in endometrial tumors. We report here the differential and increased expression of the progestin and adipoQ receptor family member 4 (PAQR4) in human endometrial cancer.

\section{Methods}

We utilized datasets GSE63678 5 and GSE115810 ${ }^{6}$ for this global differential gene expression analysis of human endometrial cancer in conjunction with GEO2R. GSE63678 was generated using Affymetrix Human Genome U133A 2.0 Array technology with $n=5$ control endometrial tissues (including $n=4$ uterine myomas and $n=1$ benign cyst) and $n=7$ endometrial cancers (including $n=2$ endometrial adenocarcinomas, $n=3$ mixed endometrioid adenocarcinomas, and $n=2$ adenocarcinomas with squamous differentiation); analysis was performed using platform GPL571. GSE115810 was generated using Affymetrix Human Genome U133A Array technology with $n=3$ control endometrial tissues and $n=24$ endometrial cancers; analysis was performed using platform GPL96. The Benjamini and Hochberg method of $p$-value adjustment was used for ranking of differential expression but raw $p$-values were used to assess statistical significance of global differential expression. Log-transformation of data was auto-detected, and the NCBI generated category of platform annotation was used. A statistical test was performed to evaluate whether PAQR4 gene expression was significantly different between control endometrial tissue and endometrial tumor tissue in humans using a two-tailed t-test. For Kaplan-Meier survival analysis, we used the Kaplan-Meier plotter tool ${ }^{7}$ for correlation of PAQR4 mRNA expression levels with overall survival in $n=543$ endometrial cancer patients.

\section{$\underline{\text { Results }}$}

We harnessed the power of blind comparative transcriptome analysis using published microarray data ${ }^{5,6}$ to discover in an unbiased fashion genes associated with endometrial cancer in humans.

\section{PAQR4 is differentially expressed in endometrial cancer.}

We identified progestin and adipoQ receptor family member 4, encoded by PAQR4, as among the genes most differentially expressed in cancers of the endometrium when compared to benign endometrial tissues (Chart 1). When sorting each of the genes expressed in endometrial tumor tissue based on significance of change in expression as compared to benign endometrial tissue, PAQR4 ranked 135 out of 22273 transcripts, equating to $99.4 \%$ differential expression (Chart 1). Differential expression of PAQR4 in human endometrial cancers was statistically significant (Chart $1 ; p=1.39 \mathrm{E}-04)$. 
We queried a second microarray data to validate differential expression of PAQR4 in endometrial cancer. Again, we observed differential expression of PAQR4 when comparing endometrial tumor tissue to benign endometrial tissue (Chart 2). When sorting each of the genes expressed in endometrial tumor tissue based on significance of change in expression as compared to benign endometrial tissue, PAQR4 ranked 8109 out of 22283 transcripts, equating to $63.6 \%$ differential expression (Chart 2). Differential expression of PAQR4 in human endometrial cancers was not deemed statistically significant (Chart $2 ; p=0.2319096$ ).

\section{PAQR4 is expressed at significantly higher levels in endometrial cancers as compared to benign endometrial tissue.}

We obtained exact mRNA expression levels for PAQR4 in endometrial tumor tissues and from benign endometrial tissue to evaluate direction and statistical significance of change in expression of PAQR4 in human endometrial cancer. PAQR4 was expressed at higher levels in endometrial tissue as compared to normal endometrial tissue, and this difference was statistically significant (Figure $1 ; p=0.0002$ ). We calculated a mean fold change of 1.21 in PAQR4 mRNA levels in human endometrial cancer, as PAQR4 was expressed at $6.09 \pm 0.28$ arbitrary units (A.U.) in control endometrial tissue but at $7.34 \pm 0.48$ A.U. in endometrial tumor tissue.

\section{PAQR4 expression is correlated with patient survival outcomes in endometrial cancer.}

We performed Kaplan-Meier survival analysis to evaluate correlation between PAQR4 primary tumor expression and survival outcomes in 543 patients with endometrial cancer. We observed a correlation between primary tumor expression of PAQR4 and overall survival in patients with endometrial cancer, in black patients with low mutational burden, in the lower survival quartile (Figure 2). PAQR4 primary tumor mRNA levels were a positive prognostic indicator in black endometrial cancer patients with low mutational burden. Black patients with low mutational burden whose primary tumors expressed low levels of PAQR4 possessed median OS of 20.33 months, while black patients with low mutational burden whose tumors expressed high levels of PAQR4 possessed median OS of 39.9 months. This difference in OS based on PAQR4 tumor expression in black patients with endometrial cancer with low mutational burden was statistically significant (Figure 2, Chart 3; logrank $p$-value: 0.036 ; hazard ratio: 0.32 (0.1-0.98)). PAQR4 primary endometrial tumor expression was not correlated with overall survival in black patients with high mutational burden (Figure 2, Chart 3; logrank $p$-value: 0.32; hazard ratio: $0.45(0.09-2.24))$, nor in white patients with high ((Figure 2, Chart 3; logrank p-value: 0.21 ; hazard ratio: $0.6(0.26-1.35))$ or low mutational burden (Figure 2, Chart 3; logrank $p$-value: 0.7 ; hazard ratio: $0.87(0.42-1.79))$.

Thus, by mining published microarray data ${ }^{5,6}$ in an unbiased and systematic fashion, we identified progestin and adipoQ receptor family member 4, encoded by PAQR4, as among the 


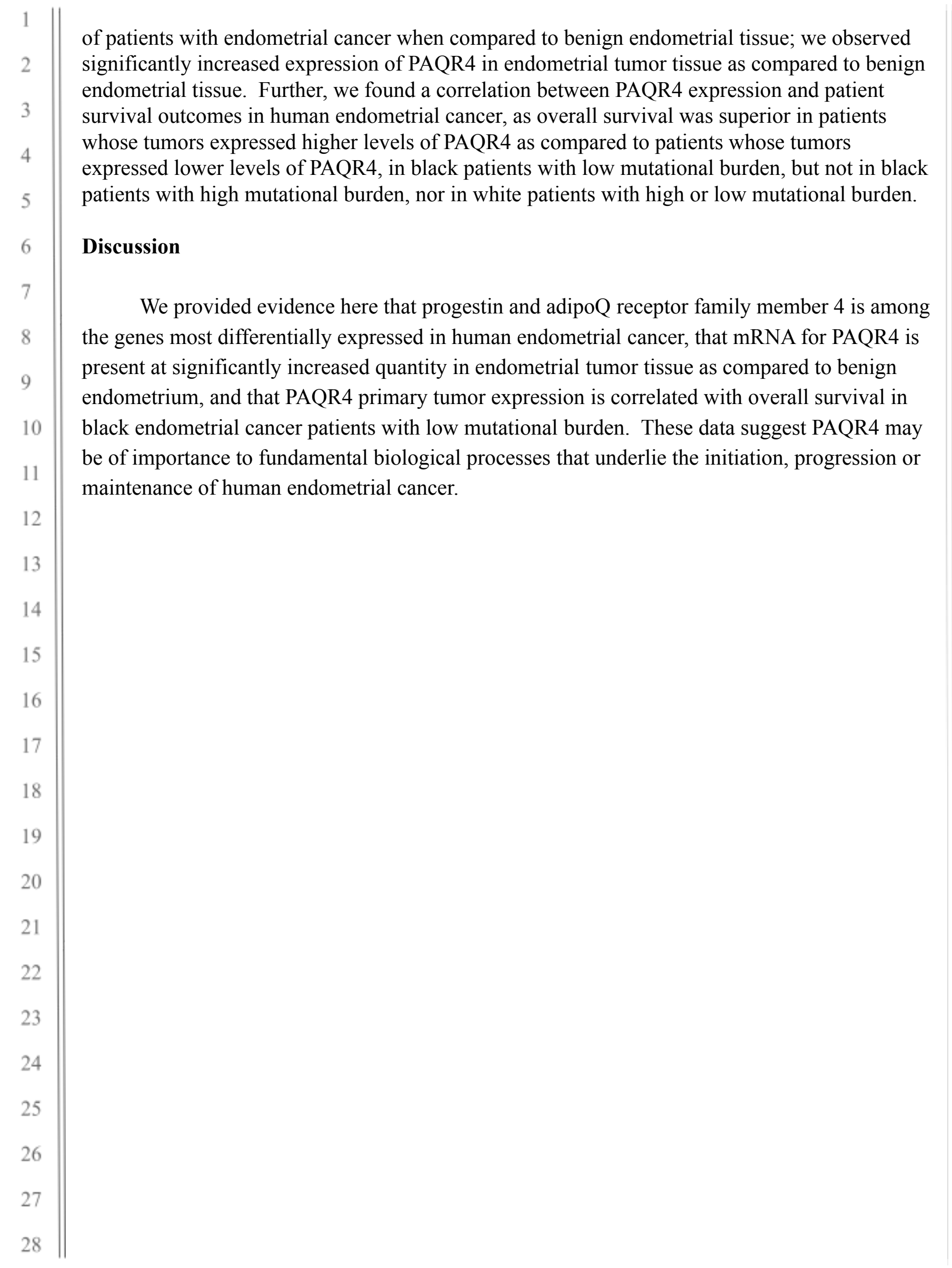




\section{References}

1. Amant, F., Moerman, P., Neven, P., Timmerman, D., Van Limbergen, E. and Vergote, I., 2005. Endometrial cancer. The Lancet, 366(9484), pp.491-505.

2. Sorosky, J.I., 2008. Endometrial cancer. Obstetrics \& Gynecology, 111(2), pp.436-447.

3. Morice, P., Leary, A., Creutzberg, C., Abu-Rustum, N. and Darai, E., 2016. Endometrial cancer. The Lancet, 387(10023), pp.1094-1108.

4. Sorosky, J.I., 2012. Endometrial cancer. Obstetrics \& Gynecology, 120(2 Part 1), pp.383-397.

5. Pappa, K.I., Polyzos, A., Jacob-Hirsch, J., Amariglio, N., Vlachos, G.D., Loutradis, D. and Anagnou, N.P., 2015. Profiling of discrete gynecological cancers reveals novel transcriptional modules and common features shared by other cancer types and embryonic stem cells. PLoS One, 10(11), p.e0142229.

6. Hermyt, E., Zmarzły, N., Grabarek, B., Kruszniewska-Rajs, C., Gola, J., Jęda-Golonka, A., Szczepanek, K., Mazurek, U. and Witek, A., 2019. Interplay between miRNAs and Genes Associated with Cell Proliferation in Endometrial Cancer. International journal of molecular sciences, 20(23), p.6011.

7. Nagy, A., Munkacsy, G. and Gyorffy, B., 2020. Pancancer survival analysis of cancer hallmark genes. bioRxiv. 
Rank: 135

Probe ID: 212858_at

p-value: $1.39 \mathrm{E}-04$

3 t: -5.4593836

4 B: 1.33868

Gene: PAQR4

Gene name: progestin and adipoQ receptor family member 4

Chart 1: PAQR4 is differentially expressed in endometrial cancer when comparing primary endometrial tumors to benign endometrial tissue.

The rank of global differential expression, probe/transcript ID, the $p$-value with respect to differential expression transcriptome-wide, $\mathrm{t}$, a moderated t-statistic, $\mathrm{B}$, the log-odds of differential expression between the groups compared, the gene and gene name are listed in this chart. 


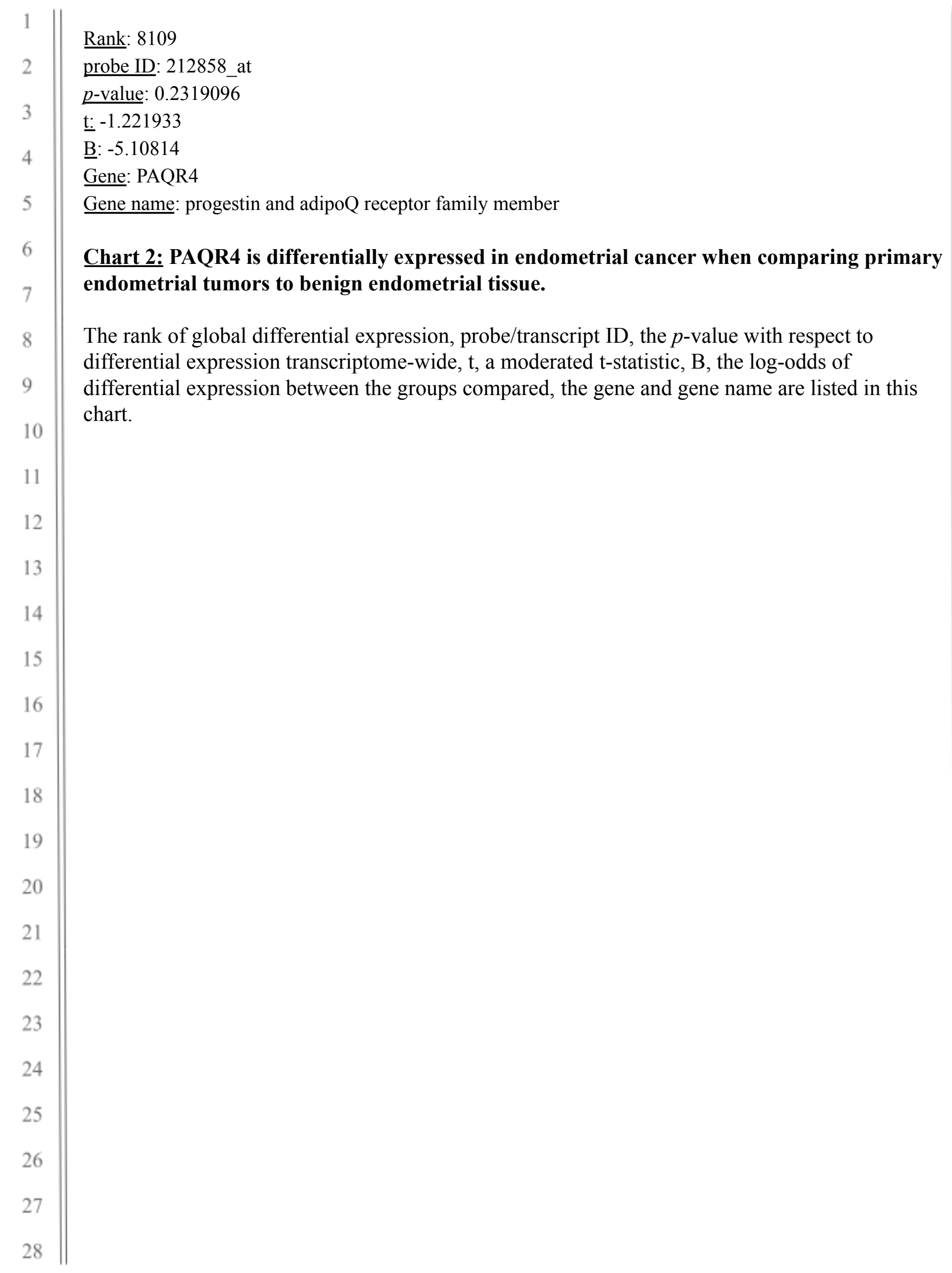




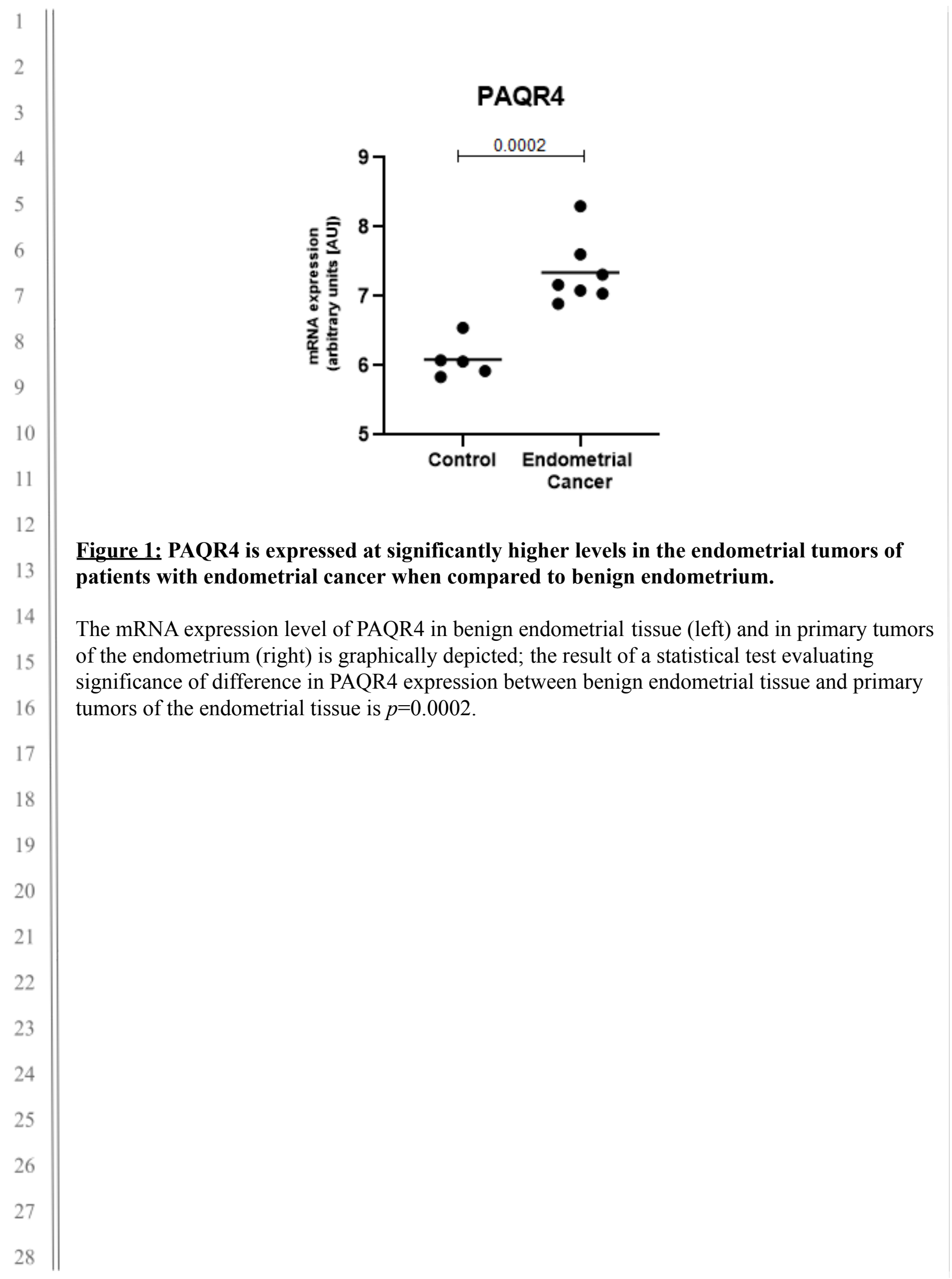




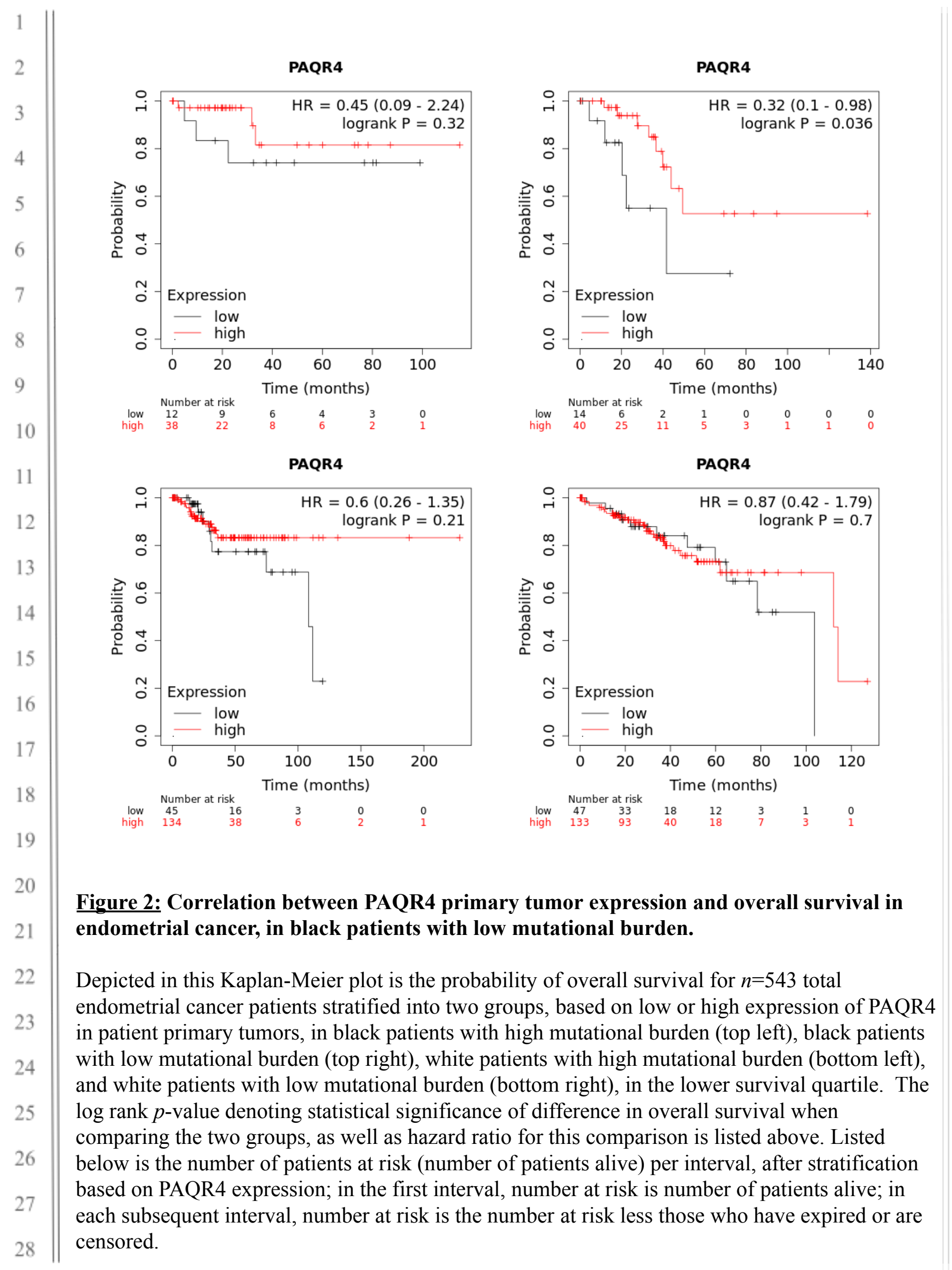


Low PAQR4 expression: 20.33 months

High PAQR4 expression: 39.9 months

Chart 3: Median overall survival is superior in endometrial cancer patients with high primary tumor expression of PAQR4, in black patients with low mutational burden.

The median OS (overall survival) of black endometrial cancer patients with low mutational burden, with low primary tumor expression of PAQR4 and high primary tumor expression of PAQR4 is listed in this chart, in the lower survival quartile. 